\title{
LARGE SCALE WAVE IMPACTS ON A VERTICAL WALL
}

\author{
Bas Hofland ${ }^{1}$, Miroslaw Lech Kaminski ${ }^{2}$ and Guido Wolters ${ }^{1}$
}

\begin{abstract}
This paper treats new large scale measurements of pressure fields on a vertical wall under wave impacts. These measurements were done in collaboration with the Joint Industry Project Sloshel, aimed at sloshing in LNG tanks. Measurements are presented with a relatively high spatial and temporal resolution. The impacts are created by wave focussing at the wall. By changing the focal point with respect to the wall, the impact type was altered. The influence of the impact type on the pressures and forces on the wall at large scale is thus studied.
\end{abstract}

Keywords: vertical wall breakwater; large scale experiments; wave impacts

\section{INTRODUCTION}

Coastal structures such as breakwaters and storm surge barriers can have vertical faces that are exposed to wave impacts. The load of wave impacts on vertical structures can be very high and can lead to structural failure. However, the process is still not very well understood (Peregrine, 2003). This paper treats new large scale measurements of pressure fields on a vertical wall under wave impacts. These measurements were done in collaboration with the Joint Industry Project Sloshel, aimed at sloshing in LNG tanks (Kaminski \& Bogaert, 2009). Note that in the context of the Sloshel project, the tests can be regarded as 'full scale', instead of 'large scale'. Only few measurements at full or large scale exist (e.g. De Rouville et al. 1938, Oumeraci et al 2001, Bullock et al 2007) with limited spatial and temporal resolution. Here large-scale measurements are presented with a high spatial and temporal resolution.

In this paper first previous research on large scale laboratory, and field situation wave impacts is described. Then the wave focussing technique that was applied in the present tests is described. Next the results are presented and analyzed. The magnitude of pressures, forces, and the repeatability of the impacts is related to the impact type (focal point). Finally the results are discussed in the light of their use for coastal engineering, and conclusions are drawn.

Figure 1. Wave impact.

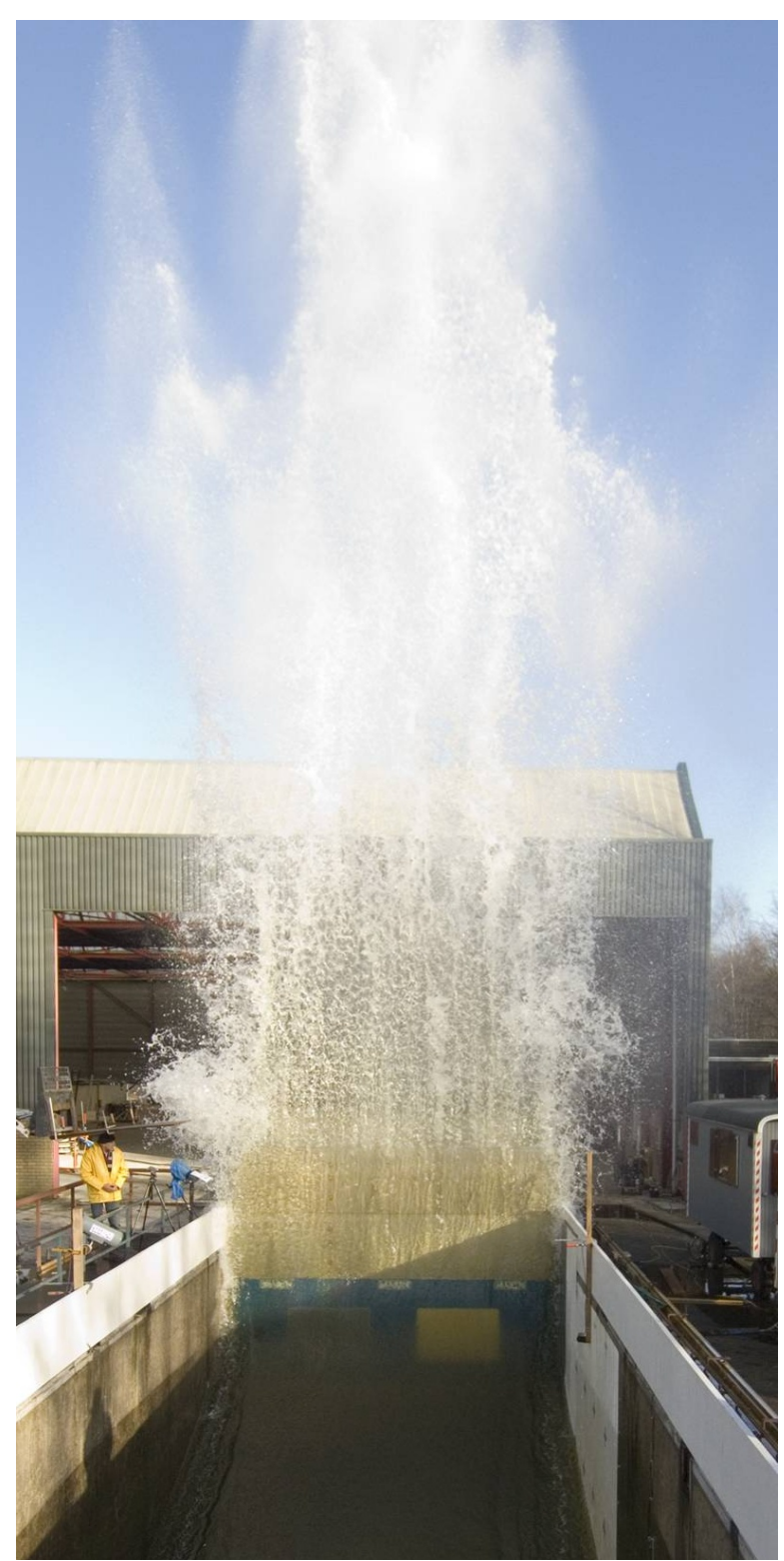

\footnotetext{
${ }_{2}^{1}$ Deltares, Hydraulic Engineering department, Delft, The Netherlands (Bas.Hofland@deltares.nl)

${ }^{2}$ MARIN, Hydro Structural Services, Wageningen, The Netherlands
} 


\section{PREVIOUS RESEARCH}

Although a large number of (small scale) laboratory tests on breaking wave impacts are documented in literature, field measurements and large scale experimental data are rare.

About twenty field studies have been carried out to date of which the authors are aware (for specifics, see e.g. Oumeraci et al. 2001). Unfortunately most of the field data which has been gathered so far is of moderate value, partly due to the limits in computing power and recording capabilities. Hence data acquisition rates (e.g. $20 \mathrm{~Hz}$ for most of the field measurements during the PROVERBS project at Dieppe, Gijon, Porto Torres, Las Palmas) were chosen which were much too low for the analysis of dynamic impact pressure events. On the other hand the instrumentation itself, apart from the logging equipment, often did not allow the measurement of pressures of very short duration in the order of milliseconds (e.g. spring dynamometers).

Field measurements of wave loading were obtained and documented as early as the 1840's by Stevenson (1874) at Dunbar harbour. The recording of the wave climate was based on visual observations and the pressure magnitude on spring dynamometers. But only since the first use of stepped wave gauges and piezo-electric transducers in 1937 by Rouville, Besson and Petry at their site in Dieppe, France, have measurements of wave climate and pressure magnitude at reasonable time scales been possible (Rouville et al. 1938). The largest impact pressure they measured was $696 \mathrm{kPa}$. The vertical pressure distributions Rouville measured demonstrate the very localized and transient nature of the dynamic wave impact pressures (variations of $\sim 90 \%$ over $1 \mathrm{~m}$ ), which was also verified by the more recent tests at Alderney. Due to their relatively good quality the results of Rouville's study were used for the calibration of various empirical models since (e.g. Bagnold 1939; Minikin 1963) and is still often quoted.

Another significant study on wave impact pressures (based on piezo-electric transducers), and associated seabed measurements of the wave climate, was done by Blackmore \& Hewson (1984) at four sites in Southern England. Here relatively small impact pressures of broken wave impacts were measured.

More recently two field investigations of wave impact pressures (and associated wave climate) were performed at the Admiralty breakwater in Alderney (Channel Islands). The first one was conducted by Bullock et al. (2000) with a very similar set up as the second study in 2004 (Bullock et al. 2004). The main difference is the more modern computer/electronic equipment used in the latter study. In Bullock et al. (2004) a data acquisition rate of $4 \mathrm{kHz}$ was used, whereas $0.5 \mathrm{kHz}$ was used in the previous study and aeration measurement techniques were employed. Recorded impact pressures reached up to $750 \mathrm{kPa}$ with an impact rise time of several milliseconds. Aeration levels recorded ranged from relatively low (7\%, for the maximum recorded impact pressure) to $25 \%$ and larger. The typical (dynamic) impact loading events were about a factor of 10 larger than previously expected, with very short pressure rise times.

Based on the Admiralty Breakwater large scale tests at a scale of 1:4 were also conducted at the large wave flume (GWK) in Hanover (Bullock et al. 2007). These fresh water tests produced much higher impact pressures than recorded in the field so far. Impact pressures up to about $3500 \mathrm{kPa}$ were recorded with rise times as short as $1.2 \mathrm{~ms}$.

Currently vertical breakwaters are most commonly designed on the basis of guidelines developed by Goda in Japan (Goda, 2000). They were originally developed for caisson breakwaters and are partly based on case studies of the sliding failure of prototype structures. Goda's method is currently used to compute the wave impact loading for non-breaking, broken and breaking waves (Takahashi extension). Goda's formula does not give actual pressures, but equivalent static loads for the dynamic system of caisson, mound and foundation. These effective loads are smaller than the applied loads.

Recently experiments were conducted at a rather large scale in the LIM flume in Barcelona (Cuomo et al. 2010). Experiments were done on wave impacts on a near vertical (10:1) wall at a steep (1:13) foreshore slope for various wave conditions and water levels. Pressure transducers were used with a rather large $20 \mathrm{~cm}$ spacing. Impact forces were obtained from pressure integration. It was found that the impact forces depended on the wave length at the structure, a parameter describing the depth at breaking compared to the local depth, and the reflection coefficient of the structure. 


\section{PHYSICAL MODEL TESTS}

This paper describes the pressure field over the entire vertical of a large vertical wall. The wall was erected for the Sloshel project to obtain measurements of full scale sloshing impacts on the flexible isolation material inside a LNG tank. To this end mainly two areas with a $1.2 \mathrm{~m}^{2}$ surface area of the wall were densely instrumented. One area was rigid and one area was made from the isolation material (containment system) to be tested. Extra pressure sensors were added over the vertical in line with the rigid part in order to obtain pressure measurements over the full height of the wall. Now the results are also useful for the evaluation of the total force of vertical-faced coastal structures.

A schematic setup was used, with a horizontal bed in front of the wall. To enable the study of different types of breaking wave impacts, a focussed wave signal has been developed, with which the type of wave impact can be steered, see Fig. 2. This resembles the small-scale setup of Chan (1994).

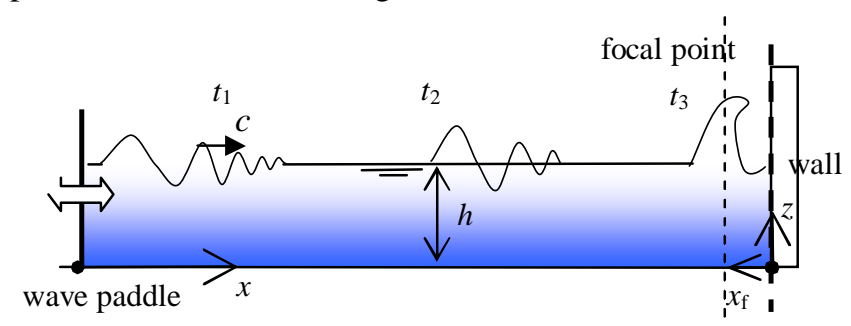

Figure 2. Schematic side view of setup and wave generation.

The tests were undertaken in the large Delta Flume of Deltares | Delft Hydraulics. This flume has a length of more than $200 \mathrm{~m}$, a depth of $7 \mathrm{~m}$ and a width of $5 \mathrm{~m}$. This flume is often used to model processes that cannot be represented at small scale but should be tested at near-full or full scale. The flume has a piston type wave generator with $5 \mathrm{~m}$ stroke, which can generate significant waves with wave heights up to $1.6 \mathrm{~m}$, regular waves of $2.5 \mathrm{~m}$ and focussed waves of over $3 \mathrm{~m}$. The Active Reflection Compensation (ARC) system on the wave paddles can absorb the wave energy that travels in the direction of the wave piston. This ARC system was used to reduce the time between impacts to less than half an hour, whereas it would otherwise have taken several hours for the flume (with vertical walls on all sides) to calm down.

Wall

A $9 \mathrm{~m}$ high wall was constructed, consisting of two $0.75 \mathrm{~m}$ thick concrete layers supported by three steel brackets. In the front wall, consisting of several concrete elements, a $2 \mathrm{~m}$ high measurement panel could be incorporated, in which much instrumentation was placed. The arrangement of the concrete elements of the front wall could be changed to accommodate tests with different sensor positions. The wall was placed at $145.2 \mathrm{~m}$ from the wave maker (middle position).
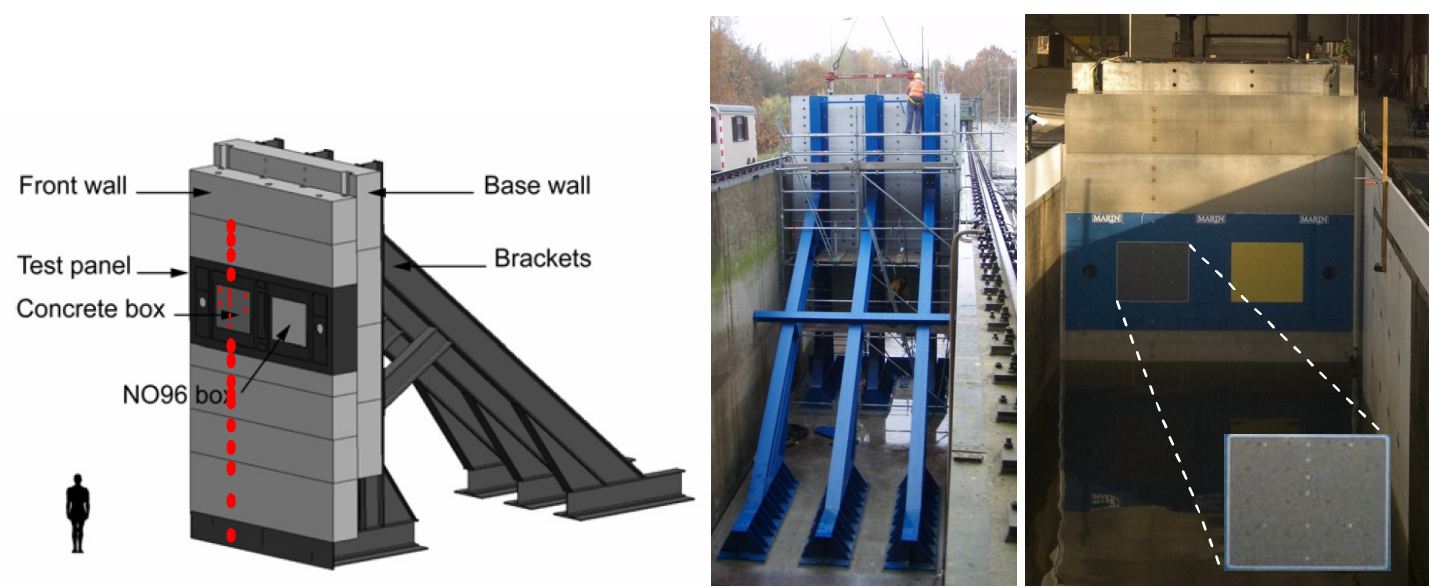

Figure 3. Test wall. Left: pressure sensors locations (red dots), middle: rear view, right: front view.

The available formulas for estimating loads on vertical walls subjected to breaking waves yielded very different loads on the wall. Therefore additional tests were conducted in the $1.2 \mathrm{~m}$ deep Scheldt flume at scale 1:6. Based on these tests the design force was specified at $6.1 \mathrm{MN}$ for a $5 \mathrm{~m}$ water 
depth. The Delta flume had to be strengthened in order to resist this large impact force and to provide sufficient support for the test wall. The design force was indeed approached as 4.1 MN maximum total force was measured at a $3.3 \mathrm{~m}$ water depth (impact s41).

\section{Measurements}

A large number measurements were made during the Sloshel project. This paper discusses only few of them, mainly those aimed at the rigid part of the wall, see the left panel of Fig. 3. Mainly wave height measurements and pressure measurements are discussed in this paper. Other measurements included measurements of water level contours on the flume wall obtained by digital (high speed) video recordings, accelerations of the wall, and the total force on two areas of $1.2 \mathrm{~m}^{2}$ on the wall. An array of many optical step-gauge type sensors mounted on the sidewall (iCam) was used to identify the breaker type in front of the structure (Kaminski \& Bogaert, 2009).

A total number of 23 pressure sensors were applied with a sampling rate of 25 or $50 \mathrm{kHz}$. The sensors were selected to fulfil a number of criteria:

- the impact can be of very short duration and, therefore, the pressure gauge should have a very high natural frequency

- the gauge experiences a thermal shock during an impact because the gauge and the supporting structure are suddenly immersed in a medium of different temperature and/or thermal conductivity

- the gauge experiences a high acceleration during an impact

- the gauge has a sensing area with finite dimensions

- the pressure is affected by vibrations of the supporting structure

- the data acquisition system may have a limited sampling rate and different filters that may change the actual pressure

The most dense spacing of pressure sensors was present in the steel measurement panel that was incorporated in the wall. Here the smallest vertical distance between pressure sensors was $12 \mathrm{~cm}$. Further away from the impact region the sensor spacing varied between $0.2 \mathrm{~m}$ and $1 \mathrm{~m}$, with the sensor spacing generally increasing away from the impact region. The miniature pressure sensors that were applied in the region where the largest pressures occur, were tailor made for these test, including a thermal isolation to remove temperature shock. They have a $3.6 \mathrm{~mm}$ diameter diaphragm. They were tested by placing them in a free falling cone that hit the water surface with a very high speed. The measured pressures were compared to a theoretical solution of the pressures on a cone that freely impacts a still water surface. The pressure sensors outside the densely instrumented area on the measurement panel were Druck sensors with a 5, 10 or 20 bar range. These sensors were coated by polyurethane to prevent the thermal shock effect. These sensors were tested with a wedge impacting a still water surface, in order to ensure their dynamic response.

\section{Wave generation}

To enable the study of different types of breaking wave impacts, a focussed wave signal has been developed, which produces a wave that propagates over a flat bed, and breaks at a designated location. An algorithm was made for the generation of the steering signal, based on the work by Van den Boomgaard (2003), with some new features added. The breaking point of the wave can be shifted explicitly. Thereby the type of wave impact can be steered without altering the setup or the water level. In Fig. 15 some breaker types are indicated, corresponding to varying focal points in front of the vertical wall, and the measured peak forces.

In most approaches that are reported on wave focussing (e.g. Chan 1990, Claus 2002) the focusing signal is developed in the frequency domain. In the present approach the signal was derived in the time domain. See Fig. 4 for an illustration of the principle of both techniques. The advantage of the present approach is that an explicit empirical non-linear dispersion relation for a regular wave can be used to determine the wave number and phase celerity from the group velocity (e.g. Kirby \& Dalrymple 1986, and subsequent discussion). Also an extension towards a model for propagation over an uneven bottom will probably also become more easy. The use of the theory is complicated by the fact that the last wave is treated separately. 
In the frequency domain approach, several sine waves that extend over the entire spatial domain, which have a peak at the focal point at the focal time, are shifted according to their own phase speed. These sines are summed in order to obtain the wave signal at the focal point.
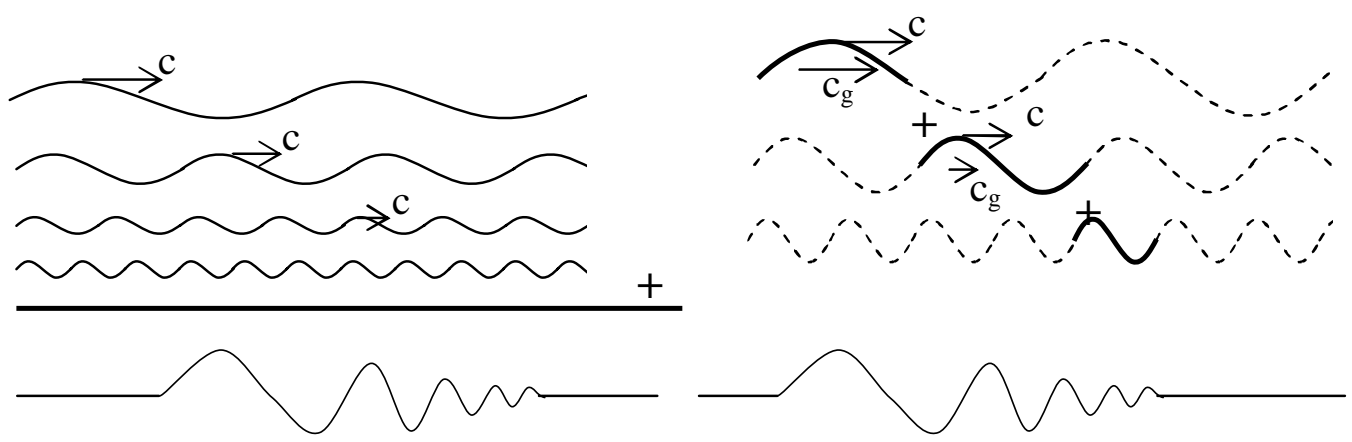

Figure 4. Focussing in frequency domain (left), and in time domain (right).

In the present approach the signal can be thought of as being composed of (very small) wave groups with their own group speed and phase speed, which both are focussed at the focal point $(x=0$ at $t=0$ ). If at each point in time the water surface elevation at the wave maker is seen as a single wave package, of which phase and energy are focussed at $x=0$ at $t=0$, a continuous expression can be obtained for a focussed wave signal at the wave maker.

$$
\begin{gathered}
\eta=a \cos \left(k(-L-c t)-\phi_{\mathrm{s}}\right) \\
c_{\mathrm{g}}=-\frac{L}{t}
\end{gathered}
$$

where $\eta$ is the surface elevation from the still water level, $a$ is the amplitude, $k$ is the wave number, $c$ is the wave celerity, $c_{\mathrm{g}}$ is the group celerity, $L$ is the distance between wave maker and focal point, and $\phi_{\mathrm{s}}$ is a phase shift. Both $k$ and $c$ follow from $c_{\mathrm{g}}$. From the water surface elevation in eq. (1) the wave paddle signal was derived by standard linear theory.

The amplitude was chosen to be a function of the wave number (which again is obtained from $\mathrm{c}_{\mathrm{g}}$ ) via an adapted Miche breaking criterion. However, in order to prevent breaking of the smaller waves before they had merged with the primary wave, a near quadratic decay of the amplitude with frequency was used, such that the leading waves (almost) did not break before the waves had merged into one large wave. In this way the amplitude distribution was tuned for a certain focal point distance. When the focal point was shifted, the amplitude was altered slightly, such that the same wave energy was generated for each wave number.

The phase shift was determined by comparing the present formula to an analytical solution by Cauchy and Poisson (see e.g. Lamb 1932). This is discussed next. Their solution of the outward propagation of linear deep water waves due to an initial disturbance of the water surface is compared to the present method. To this end the standard wave celerities of linear deep water waves $(c=\sqrt{ }(g / k)$ and $c_{\mathrm{g}}=1 / 2 c$ ) are substituted in eq. (1):

$$
\eta=a(k) \cos \left(-\frac{g t^{2}}{4 L}-\phi_{\mathrm{s}}\right)
$$

Cauchy and Poisson derived the analytical solution for waves moving outward from an initial condition with still water and a surface elevation shaped as a delta pulse at $\mathrm{x}=0, \mathrm{t}=0$. This is the reverse problem as wave focussing, as waves travel away from a single point, instead of towards it. If we write their solution in the form of eq. (2), and we use a negative time, as in the focussing case the wave travels to the other direction, it follows that for the far-field (i.e. large values of $\left|\mathrm{g} t^{2} / 4 L\right|$ ) this yields: 


$$
\eta=\frac{\sqrt{g} t}{2 \sqrt{2 \pi} L^{3 / 2}} \cos \left(-\frac{g t^{2}}{4 L}+\frac{3}{4} \pi\right)
$$

Except for the amplitude, this is equal to the present relation (2) with $\phi_{\mathrm{s}}=-3 / 4 \pi$. This is the value of $\phi_{\mathrm{s}}$ that was used for creating the wave impacts. The value can be changed slightly in order to alter the wave shape.

From the solution above it appears that the signal of eq. (1) might not be valid anymore for the last part, this is indicated schematically in Fig. 5, where the propagation of the energy is depicted in an $\mathrm{x}$-t diagram. For this reason, and to ensure a gradual transition of the last wave to a still water surface, the part of the wave maker signal later than the last zero-upcrossing was replaced by half of the wave maker signal for creating a soliton (Goring and Raichlen 1980). The height of the soliton was determined such that the maximum stroke of the Delta Flume wave maker would be used at a water level of $\mathrm{h}=5 \mathrm{~m}$.

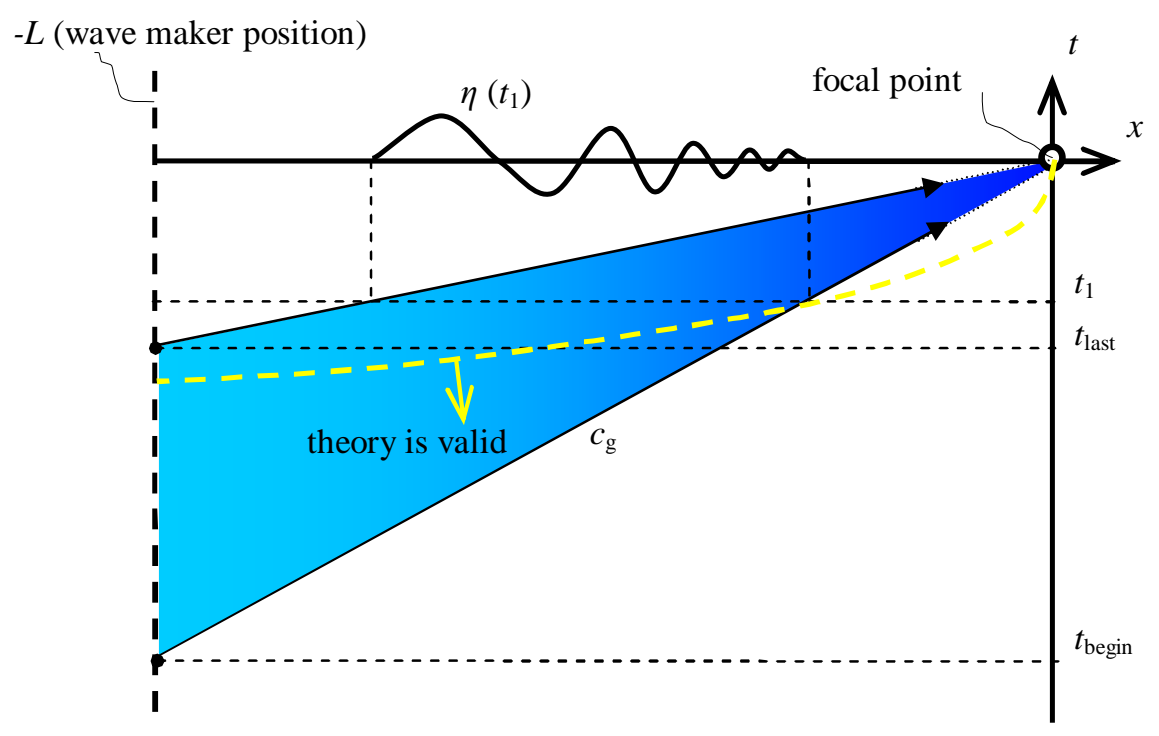

Figure 5. Focussing energy. The generated wave train is located in the shaded area of the x-t plane.

During the tests all wave parameters were kept constant, except for the exact focal distance L. This value was increased or decreased by the value of $x_{\mathrm{f}}$, the distance of the wall to the focal point. This was the main parameter that was used to create different types of impact. If another water level is used, the entire wave signal is adapted by Froude scaling. In this case the dimensionless distance between wave maker and wall has altered, such that the focal point has to be corrected for this. This extra focal point shift to correct for a different water level is much larger than the shift needed to obtain another impact type.

The last wave of the wave group increases in height when travelling towards the focal point. It reaches a maximum height of slightly over $0.6 h$ just before the focal point, after which the wave starts breaking, see Fig. 6.
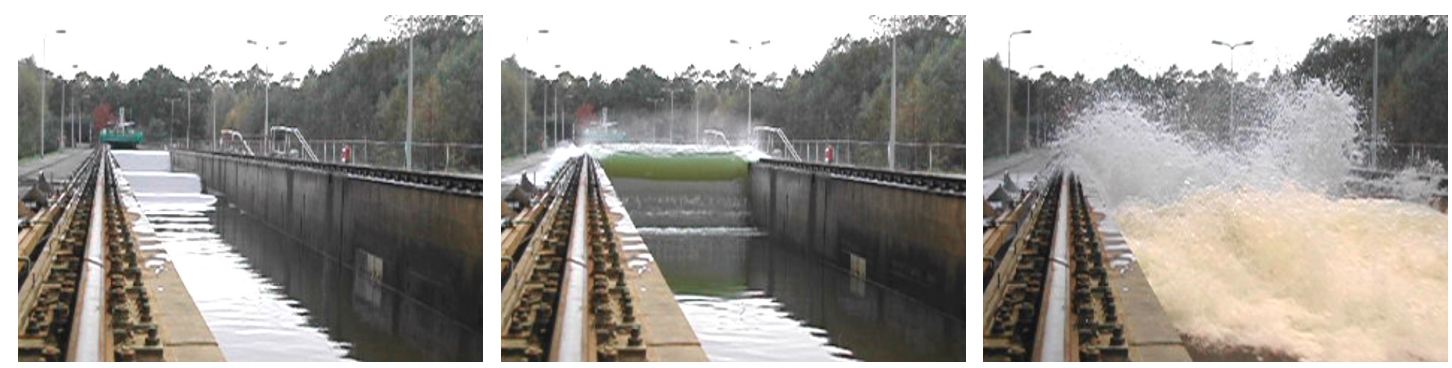

Figure 6. Focussed wave with $\mathrm{H}_{\max }=\mathbf{3 . 0 5} \mathrm{m}$ in de Delta Flume. 


\section{Test programme}

In total 137 impacts were measured with water depths varying from $h=2.5$ to $h=4.25 \mathrm{~m}$. The arrangement of the concrete elements of the front wall was changed to accommodate tests with different water depths/sensor positions. Three arrangements were used. In the first configuration no additional measurement panel was present (tests T001-T027) and water depths of 2.5 and $3.5 \mathrm{~m}$ were applied. The second configuration had the densest instrumented areas situated between 4 and $5 \mathrm{~m}$ from the flume bed and water depths of 3.3 and $3.5 \mathrm{~m}$ were applied (tests S001-S047). The third configuration had the densest instrumented area situated between 5 and $6 \mathrm{~m}$ from the flume bed and water depths of 4.25 and $4.0 \mathrm{~m}$ were applied. The total height of the front wall was $8.5 \mathrm{~m}$ in the second and third configurations.

The wave signal was the same for all tests, except for the shift of the focal point, with a maximum wave height of $0.6 \mathrm{~h}$. The focal point was altered between tests to obtain different impact types. Many tests were executed at focal points near the value needed to obtain a flip through impact, as about 10 repetitions were needed to obtain one very violent impact

\section{RESULTS}

\section{Wave field}

When the water depth is changed, the relative distance between wave maker and wall (measured in number of water depths) is increased considerably. Therefore the focal point has to be shifted to obtain the same kind of impact. It became clear that even after this extra shift of the focal point, the impact type was not the same for different water levels. Therefore the focal points were corrected empirically (by a function of water depth) such that on average the same kind of impact occurred at the same value of this corrected focal point. However, the wave created for a water depth of $h=2.5$ was different from the wave created for water levels in the range of $3.3-4.25 \mathrm{~m}$. Both the type of impact (wave height) and response (pressures) changed. Therefore the results of these tests are indicated by light blue markers in the coming figures, e.g. Figs. 7, 10, 11 and 15. This might be caused by the ad-hoc way that the soliton signal was 'pasted' to the chirp-signal.
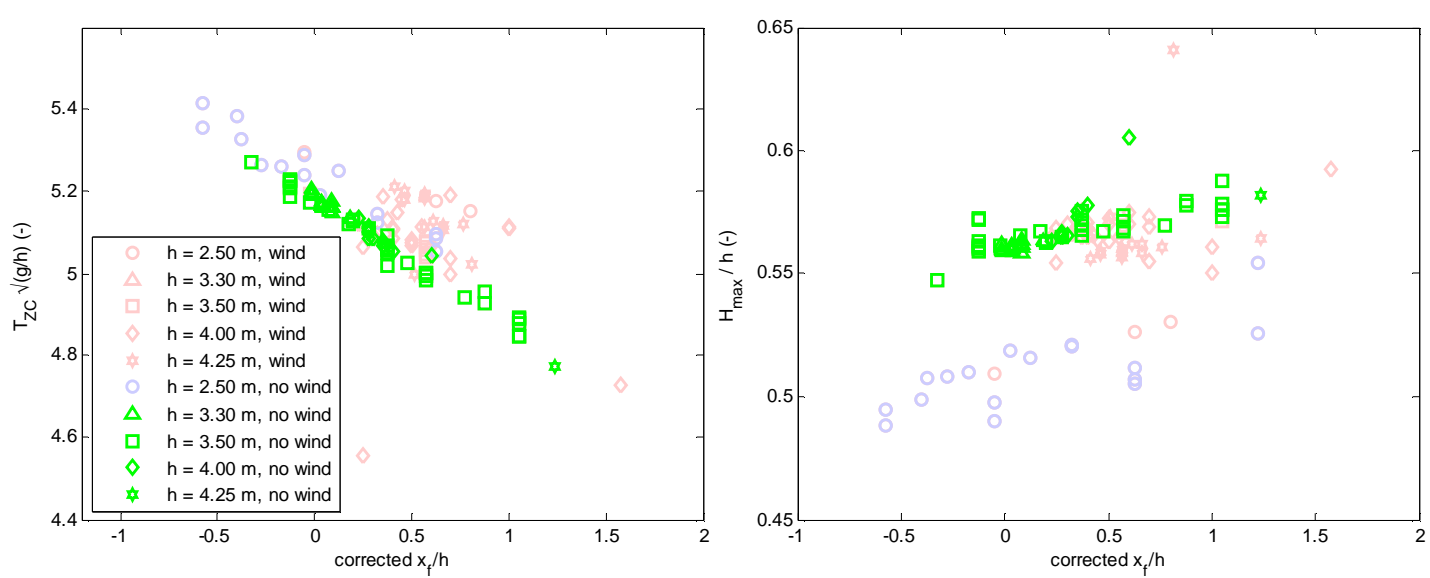

Figure 7. Variation of dimensionless maximum up-crossing period (left) and wave height measured at $4 \mathrm{~h}$ from wall.

In Fig. 7 it can be seen that the zero-crossing period of the primary wave, $T_{\mathrm{zc}}$, decreases, and its height increases, as the wave comes closer to the focal point $x_{\mathrm{f}}$. The waves are measured at a fixed location of $4 h$ with respect to the wall, so with increasing distance wall-focal point, $x_{\mathrm{f}}$, the focal point comes closer to the wave measurement.

\section{Impact types}

Several types of impact have been identified previously (see e.g. Oumeraci et al 1993, Hull \& Müller 2002, Bullock et al 2007). It was possible to obtain several types of impact that resemble those mentioned in literature during the tests by changing the focal point of the wave. These are: 
- The aerated impact, where the wave has been broken and an aerated water mass hits the wall, this impact occurs for waves that break far in front of the wall.

- The air pocket impact, where at the moment of impact the wave crest hits the wall, and wall and wave enclose a semi-circular air bubble, see Fig. 9. This kind of impact occurs in a range of focal points of roughly $0.7 h$.

- The flip through impact, where the wave trough and wave crest meet at the moment of impact such that (almost) all air has been expelled from the air pocket at the moment of impact (e.g. Peregrine, 2003), see Fig. 8. It became clear that a flip through impact occurred in a narrow range of focal points of less than $0.05 \mathrm{~h}$.

- The slosh impact, where the runup of the wave is higher than the wave crest, so that the wave crest hits the water layer instead of the wall. This kind of impact occurs for waves that would break far behind the wall.

These impact types can (for the given set of generation parameters) be defined by a certain range of focal points. However, within this range of focal points the impact shape and related loads will also alter. For instance if the focal point moves further from the wall, the air pocket size of an air pocket type impact will generally increase, and therefore the vibration frequency of pressures will alter. We will present the measured forces and pressures as a function of the focal point, which can be regarded as a parameter describing the wave shape.
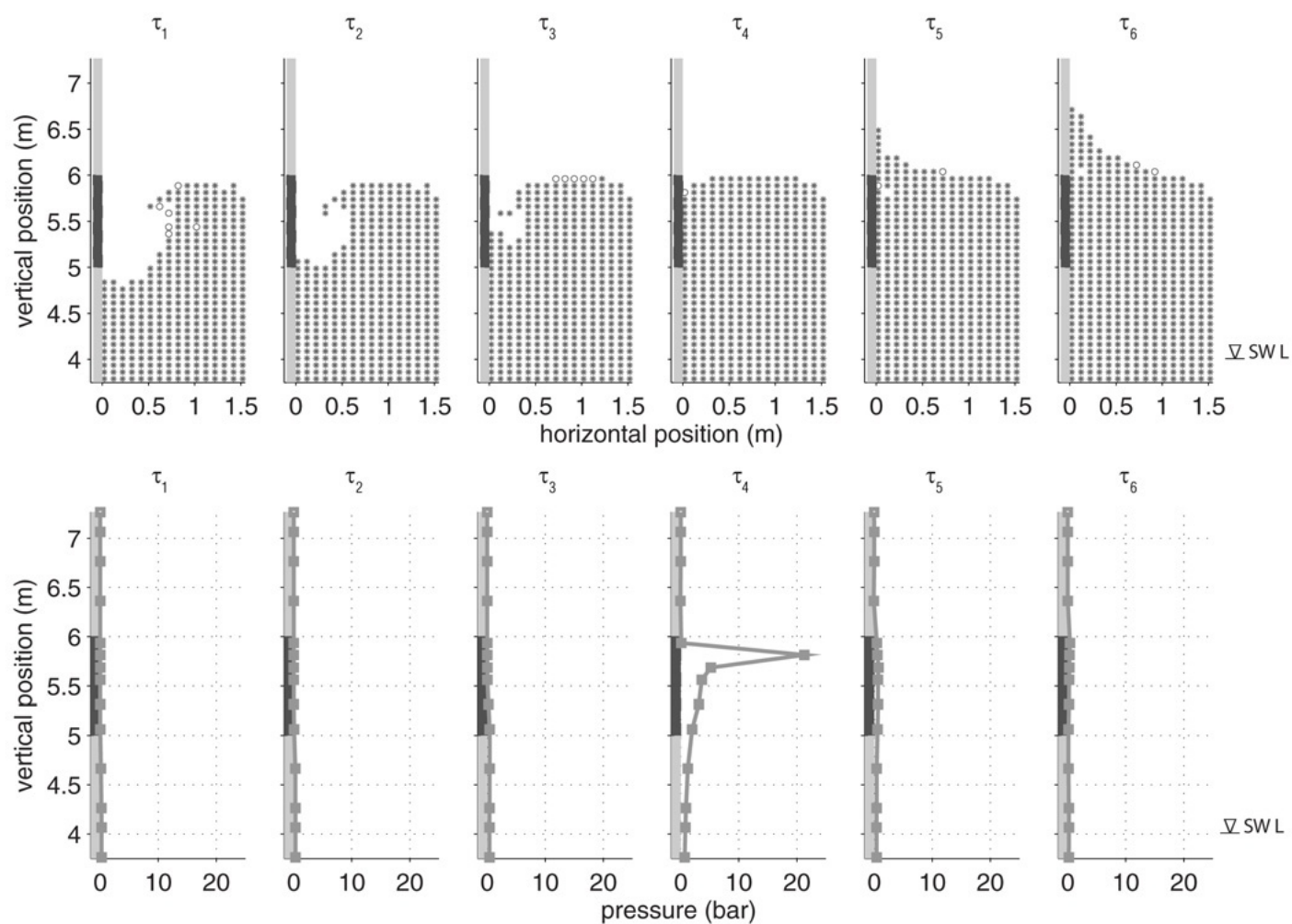

Figure 8. Flip through impact (no. S74) (Kaminski \& Bogaert, 2009). 

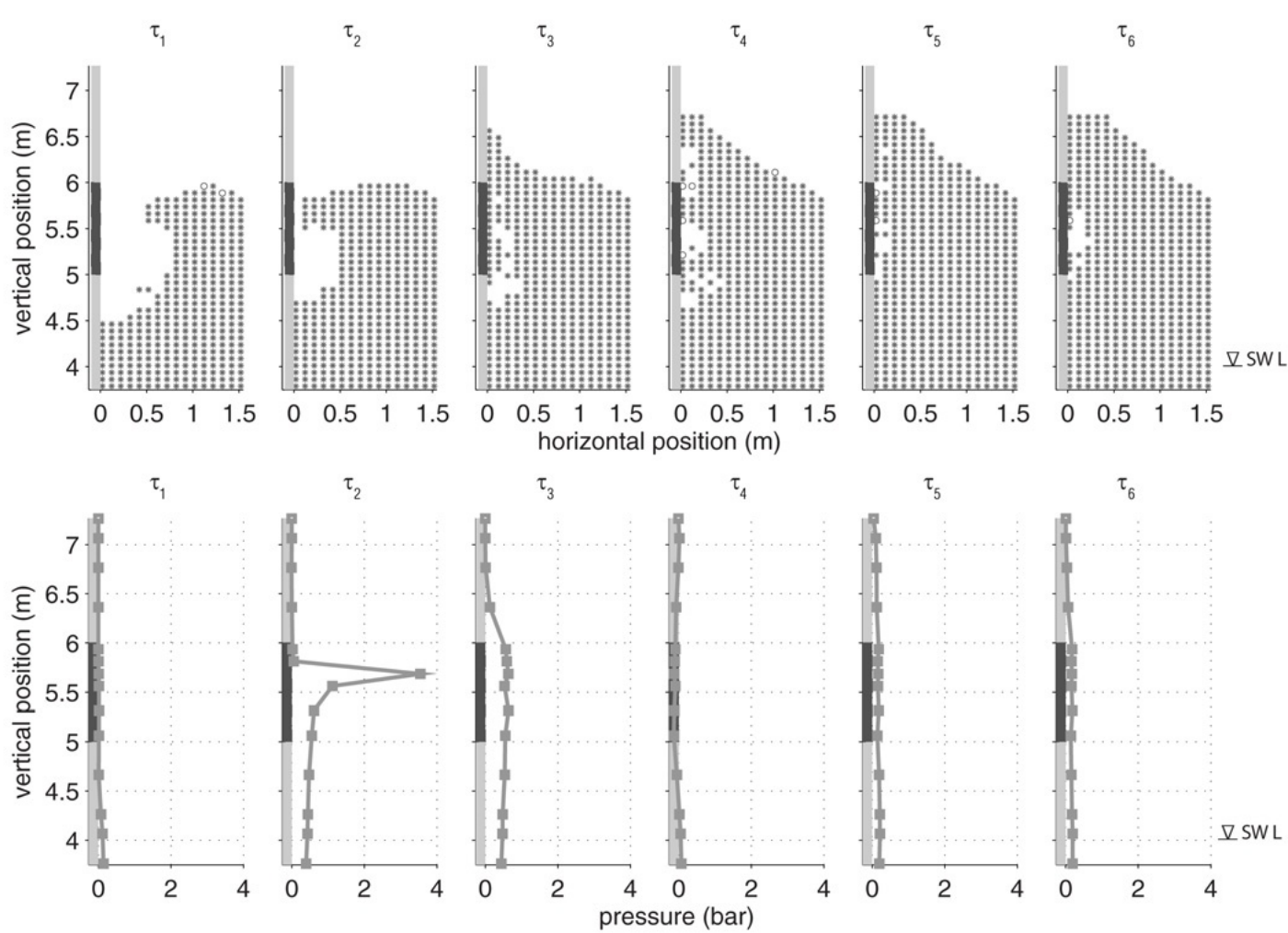

$\begin{array}{llll}0 & 0.5 & 1 & 1.5\end{array}$

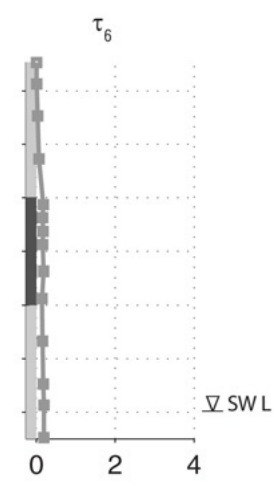

Figure 9. Air pocket impact (no. S79) (Kaminski \& Bogaert, 2009).

\section{Validity of force integration}

From the present tests a time history of the total force has been obtained by integration of pressures. As especially the highest pressures can have spatially very limited dimensions, the force integration was checked by comparing the integrated pressures over the densely instrumented $1.2 \mathrm{~m}^{2}$ area to a force measurement that was made simultaneously over this entire area. This was analyzed for the 10 impacts with the largest forces (impacts S35, S41, S40, S99, S33, S107, S037, S110, S097, and S100). Generally a good agreement was found.

Both pressure measurement and force measurement can have some drawbacks. For some of the large impacts the integrated-pressure force show a double force peak. This can be caused by the fact that a peak pressure with a spatial scale smaller than the pressure sensors spacing $(12 \mathrm{~cm})$ advected over two pressure sensors consecutively. This effect will be most pronounced in the $1.2 \mathrm{~m}^{2}$ area that is compared. However, when the pressure profile was rather smooth, the direct force measurement could overestimate the force as some dynamic amplification might have influenced the measurements. It seemed that the direct force measurement only captured variations in time larger than a few ms.

If the integrated-pressure force signal was filtered by a moving average of $2 \mathrm{~ms}$ it resembled the directly measured force best (no bias). Therefore all forces were filtered in that manner. Now the difference between the filtered force and force plate measurements were about $\pm 0.7 \operatorname{\rho gh}^{2} \mathrm{~B}$ (95\% confidence limits), which is regarded to be the accuracy of the total force measurement.

\section{Variability}

The analysis of video observations taken during the tests revealed that the impacts were not repeatable because of several causes:

- the effect of the wind on wave propagation (the Delta Flume is situated in open air),

- a small leading wave either breaking or reflecting just before the primary wave,

- the wave crest of the impacting wave could be quite unstable,

- small water surface perturbations in the flume. 
In later tests (not described here) some of these causes could be augmented. The flume was covered to reduce the wind over the water surface. This cover should be flexible and far from the wave crest in order not to disturb the air flow that is caused by the wave itself. By making the small leading waves in the wave train smaller, the last leading wave was altered such that this did not break anymore.

However, for the present data we have to cope with the large variability. For this we can omit the measurements where too much wind was present. This was determined by using the high frequency (f $>2 \mathrm{~Hz}$ ) wave height $\mathrm{H}_{\mathrm{s} \text {,high }}$ measured in the flume during wave generation, and the wind speed measured at an elevation of $10 \mathrm{~m}\left(\mathrm{U}_{10}\right)$. A measurement is considered not to be influenced by wind when: $\mathrm{H}_{\mathrm{s} \text {,high }}<0.008 \mathrm{~m}$ and $\mathrm{U}_{10}<2.3 \mathrm{~m} / \mathrm{s}$. The measurements of the impacts that are influenced by wind according to this criterion are indicated by a light red colour in the figures treating the trends of several parameters and focal point (Figs. 7, 10, 11, and 15).

\section{Pressures, forces, and impulse}

The variation of peak pressure $P_{\max \text { max }}$ with focal point is shown in Figs. 10 and 11 (with and without dimension). It can be seen that the variation in pressures is larger than the influence of scale (in the small range of water depths that were applied), as the scatter in the dimensionless plot is still very high.

The largest pressures were created by the flip through impacts (corrected $x_{\mathrm{f}} / h \approx 0.3$ ). Eight impacts had pressures over $1 \mathrm{MPa}(10 \mathrm{bar})$. However, many impacts with the same focal point showed much lower pressures. Most of the highest pressures peaks had a duration of less than a millisecond. However, some had a duration of about $10 \mathrm{~ms}$. The largest peak pressure measured was $2.7 \mathrm{MPa}$, with a very a short duration $(<0.5 \mathrm{~ms})$. This is comparable to the $3.5 \mathrm{MPa}$ peak pressure measured by Bullock et al (2007). The very high pressure peaks are very localized in the vertical direction. However, each time that a high peak pressure was measured, peak pressures of the same order of magnitude were measured at the measurement location at the other side of the wall (the area with the isolation system of an LNG tank). Therefore it is concluded that the pressure field was rather two dimensional.

The air pocket impacts (corrected $x_{\mathrm{f}} / h \approx 0.4-1$ ) show pressures in the range of $0.2-0.5 \mathrm{MPa}$, with a tendency of higher pressures for smaller air pockets.

The aerated (broken) wave impacts (corrected $x_{\mathrm{f}} / h>1$ ) and the slosh impacts (corrected $x_{\mathrm{f}} / h<$ 0.3) show quasi-static pressures, in the order of the wave height.

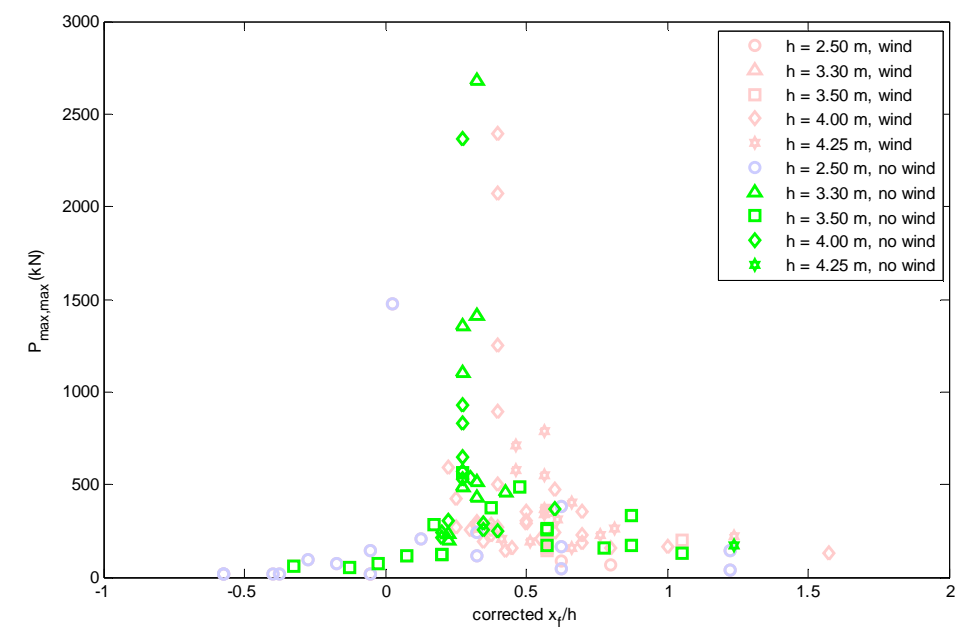

Figure 10. Measured peak pressures. 


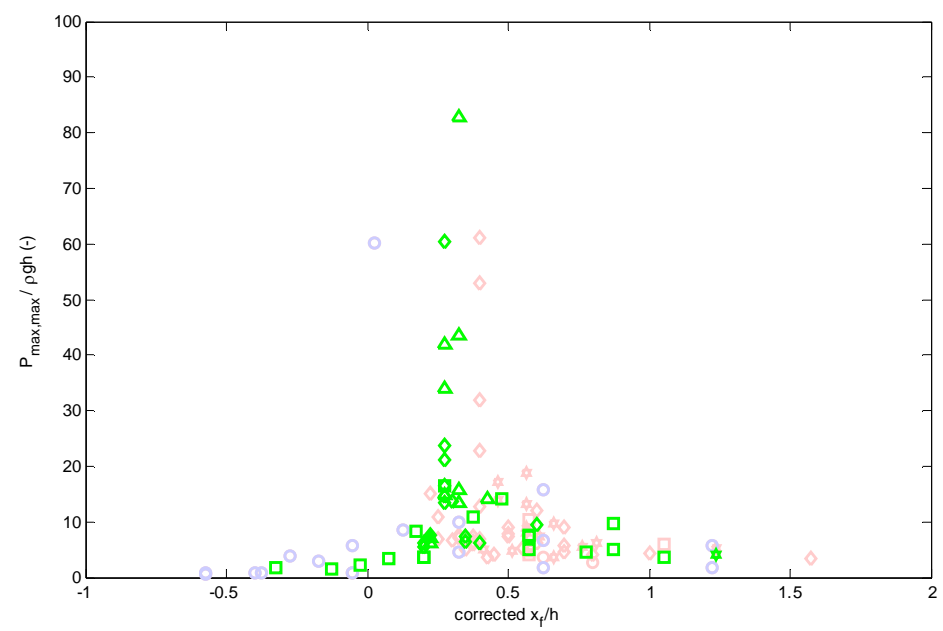

Figure 11. Variation of dimensionless peak pressures with focal point.

Due to the relatively high spatial resolution, instantaneous vertical pressure profiles can be presented, instead of profiles of separate peak pressures, see Fig. 12. The air pocket impact shows a broad, or even a double peak. The peak pressure is located at the location of the jet impact. The flip through impacts exhibit a single sharp peak at the location where the wave crest and wave trough meet.
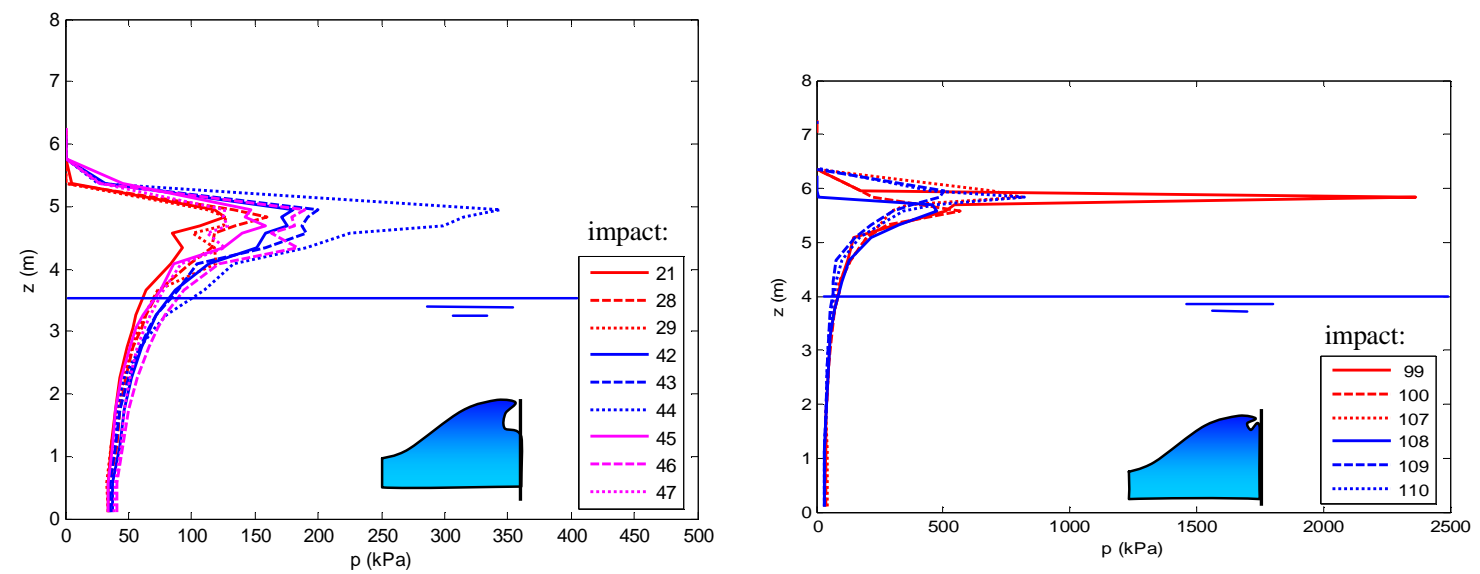

Figure 12. Instantaneous vertical pressure profiles at maximum force for air pocket (left) and flip through impacts.

The largest forces are created by the flip through impacts. The largest peak force (filtered by a 2 ms moving average) was $822 \mathrm{kN} / \mathrm{m}$ during impact s41. Most large impacts had a duration of about 10 ms. In Fig. 13 two flip through impact time traces are shown (not filtered). The oscillation after the impact corresponds to the lowest natural frequency of the wall $(12-14 \mathrm{~Hz}$, depending on water level and panel position). 

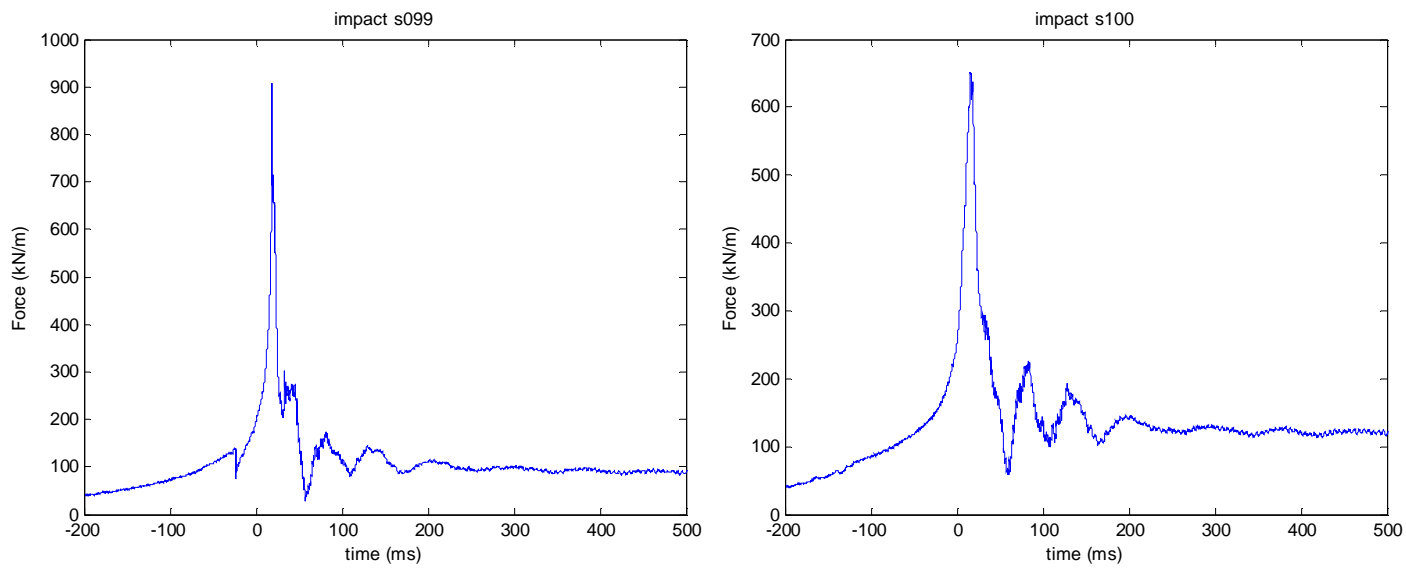

Figure 13. Forces obtained from integration of pressure profiles for 'identical' flip through impacts.

Two force plots of air pocket impacts are given in Fig. 14. The maximum forces are much lower, but the duration is longer, as could be expected, as the impulse should be constant. The lower frequency oscillation of the impact in the left plot is probably due to oscillation of the air bubble. The duration of the air pocket impacts was in the order of 30-90 ms.
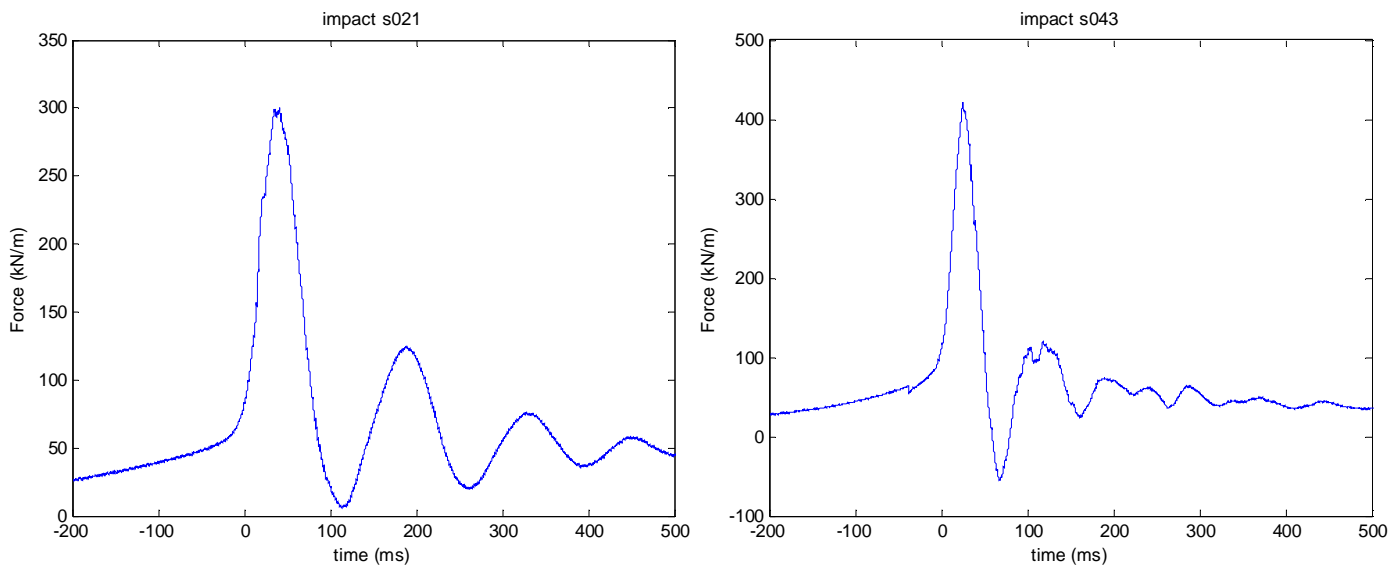

Figure 14. Forces obtained from integration of pressure profiles for 'identical' air pocket impacts.

In Fig. 15 the selected forces are given as a function of corrected focal point. It can be seen that the peak force of the impacts that are not influence by wind is a rather continuous function of focal point with a large variation where the flip through impacts occur. For the broken wave impacts at large focal points, impact forces of about $1 \rho g h^{2} B$ were measured ( $B$ is the width considered). For decreasing focal points air pocket impacts occur. The force rises gradually from about $2 \rho g h^{2} B$ to $4 \rho g h^{2} B$ as the air pocket becomes smaller. The forces can be very high when flip through impacts occur for a small range of focal points where the air pocket just closes at impact. The largest forces are 7-8 $\rho g h^{2} B$, which is equal to $19-22 \rho g H_{\mathrm{b}}{ }^{2} B$, where $H_{\mathrm{b}}$ is the wave height at breaking. For even smaller focal points slosh impacts occur and the measured peak force suddenly decreases. Here the water runup against the wall damps the wave impact. 


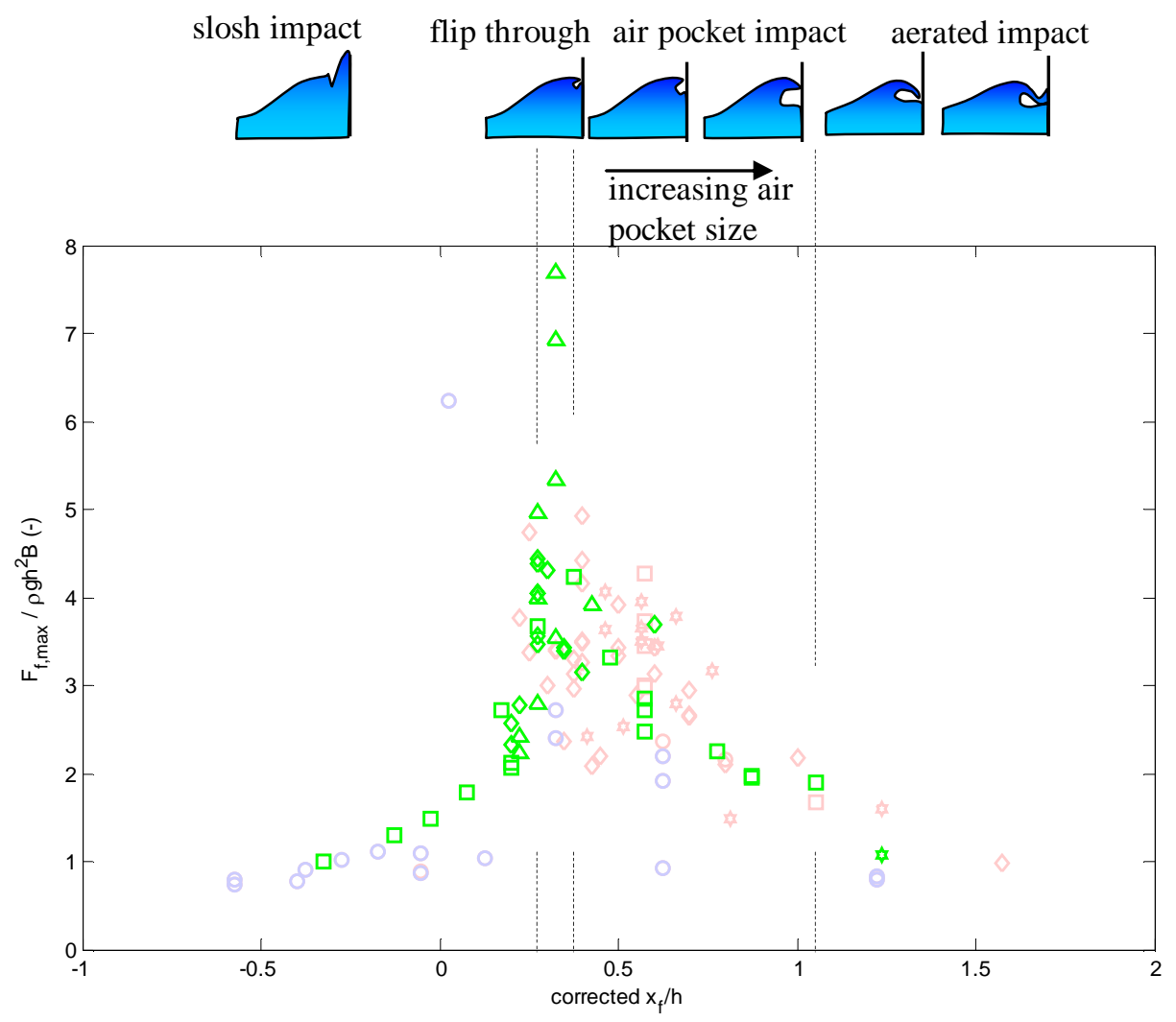

Figure 15. Variation of dimensionless force with focal point.

\section{Impact Location}

The location of the pressure peak is located above the water level for all impact types investigated, around 1.4 times the water depth. This is not in line with e.g. Goda (2000), but already noted for deep water waves (Chan 1994). The position of the peak pressure increases somewhat with a focal point that lies further from the wave maker, as the water is pushed more unto the wall. The 'flip through' impacts have the maximum pressure at $z / h=1.44$, whereas the 'air pocket' impacts have their maximum at $z / h=1.39$, where $z$ is the elevation above the flume bottom. The aerated impacts still have their peak pressure around $z=1.25 \mathrm{~h}$.

\section{Model}

The pressure-impulse theory of Cooker \& Peregrine (1995) was compared to the two series of selected impacts with air pocket and flip through types of impact. The following values for the various parameters were chosen to fit the distribution:

$H=h+\eta_{\max }$ (highest measured crest level of the wave with respect to bed),

$\mathrm{U}_{0} \quad=\sqrt{\mathrm{gH}}($ phase velocity of impacting wave),

$b \quad=\mu H$ (length of wave in $x$-direction),

$\mu \quad=0.24$ for air pocket impacts and resp. 0.03 and 0.12 for flip through impacts,

$\Delta t=$ resp. 30 and $90 \mathrm{~ms}$ for air pocket impacts and resp. 0.5 and $15 \mathrm{~ms}$ for flip through impacts (durations obtained from measured force peak)

In Fig. 16 the earlier presented measured pressure distributions for air pocket and flip through impacts are compared to the model. For the air pocket impacts the distributions for the pressure peak roughly matches the measurements. The lower elevations of the pressure profiles are caused by the pulsating or quasi-hydrostatic pressures which are not part of the impact theory. The change in pressure magnitude is explained well by the change in impact-duration. The air pocket impacts with 
high pressures had a duration of around $30 \mathrm{~ms}$, and those with low pressures had a duration around $90 \mathrm{~ms}$. Using these values to translate the pressure impulse to a pressure, a rough approximation of both the upper and lower envelope is obtained.

To fit the range of pressure profiles of the flip through impacts, not only the measured duration has to be applied, but two different values of $\mu$. This indicates that the larger impact pressures are not only related to smaller durations, but also to smaller impact areas.
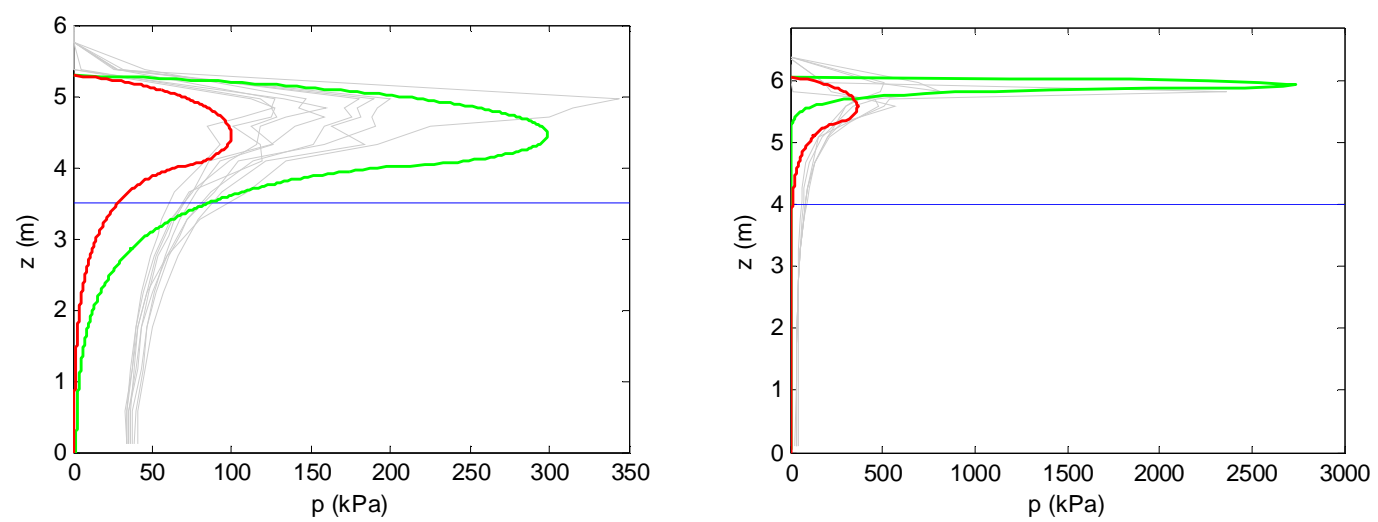

Figure 16. Measured pressure profiles (gray lines) for air pocket impacts (left) and flip through impacts, including theoretical models for wave impact (Cooker \& Peregrine 1995, red and green lines).

\section{DISCUSSION}

The fact that the variability of the impact pressures and forces is high was already noticed in the use of solitons and regular waves in previous studies (e.g. Bagnold 1939). Some causes of variability have been identified. Still it is expected that the impacts - especially the flip through impact, where the pressure field depends on small scale kinematics of the impacting wave - will also be very variable when these causes are removed.

The present method of generating the impacts can also be used to identify the probability of occurrence of certain types of impact. If the range of focal points is regarded over which the flip through impact occurs (roughly $0.05 h$ ), compared to the air pocket (roughly $0.7 h$ ), it can be seen that the probability of occurrence of the flip through impact is much lower. And even if the focal point has the right value, not every time a very violent flip through impact occurs. Moreover, in a real three dimensional situation it is unclear how often both types of impact will occur.

The high position above the water line of the impact pressure of about $0.4 h$ or $0.6-0.7 H_{\mathrm{b}}$ above SWL is mentioned in some sources but it is not clearly present in most guidelines or engineering formulas, which usually are intended for caisson breakwaters on relatively high mounds. For example, also Hull \& Müller (2002) saw peak pressures of the flip through waves far above the water line, but their air pocket peak pressures were located around SWL. Presently both impact types were found to be above the water line. If the peak impact pressure can occur much higher and with high magnitude, this can be important, for instance when designing a parapet wall on a caisson. This is connected stiffly to the concrete caisson, so the short duration impacts with high pressures might fully act on the connection of these walls.

The present set up had a very large scale, but does not resemble reality in the sense that fresh water was used. The compressibility of the air, which plays a role in the air pocket impacts has scale effects. The flip through impact probably has less scale effects except for the stiffness of the structure. The maximum pressures that were measured seem to approach the water hammer pressure, so for these impacts it seems reasonable to scale the pressure with Froude, and maximize the pressures with the water hammer pressure. The water hammer pressure itself is influenced by the air content in the water.

\section{CONCLUSIONS}

A method for wave focussing was developed for creating large scale impacts in the Delta flume. Waves with maximum heights of up to $2.6 \mathrm{~m}$ height were used to make several types of impacts on a vertical wall. The wave shape and pressure distribution were measured at large scale. 
The variability of the forces, and especially of the pressures was large. The variability was largest for the flip through impacts. The relation between wave focus location, impact type and impact load was presented. It could be seen that the flip through impacts only occurred at a very small range of focal points, making them rare in a random wave field. The flip through impacts created the largest peak pressures and peak forces. Also the air pocket impact created large forces. The high location of the peak pressure for all impact types of impacts is remarkable.

\section{ACKNOWLEDGMENTS}

The tests were done in cooperation with the Joint Industry Project Sloshel, which was funded by GTT, Marin, Bureau Veritas, Lloyd's register, ABS, DNV, ClassNK, Shell, Chevron, and Ecole Centrale Marseille. Ap van Dongeren is thanked for his significant contribution in creating the focussed wave signal.

\section{REFERENCES}

Bagnold, R.A., Interim Report on Wave Research, Journal Inst. Civ. Eng., 12, 202-226, 1938/1939

Blackmore P.A. \& Hewson P., Experiments on full-scale wave impact pressures, Coastal Engineering, 8, 331-346, 1984

Bullock G.N., Hewson P., Crawford A.R., Bird P.A.D., 2000. Field and laboratory measurements of wave loads on vertical breakwaters, Proc. Coastal Structures 99, Santander/Spain, 613-621

Bullock G., Obhrai C., Wolters G., Müller G., Peregrine H. \& Bredmose H., 2004, Characteristics and design implications of breaking wave impacts, Proc. Int. Conf. Coastal Engineering, Lisbon.

Bullock, G.N., C. Obhrai, D.H. Peregrine, H. Bredmose. 2007. Violent breaking wave impacts. Part 1: Results from large-scale regular wave tests on vertical and sloping walls. Coastal Engineering 54. 602-617

Chan, E.S. 1994. Mechanics of deep water plunging-wave impacts on vertical structures. Coastal Engineering, 22. p 115-133

Clauss, G.F. 2002. Dramas of the sea: episodic waves and their impact on offshore structures. Applied Ocean Research 24, 147-161

Cooker M.J. \& Peregrine D.H., 1995. Pressure-Impulse theory for liquid impact problems, J. Fluid Mech., 297, 193-214.

Cuomo, G., Allsop, W., Bruce, T., Pearson, J. 2010. Breaking wave loads at vertical seawalls and breakwaters. Coastal Engineering 57. 424-439

De Rouville, A., Besson, P. and Petry, P., 1938. Etat actuel des études intemationales sur les efforts dus aux lames. Ann. Ponts Chaussées, 108(I1): 5-113.

Goda Y., 2000, Random Seas and Design of Maritime Structures, World Scientific Publ., Singapore, $443 \mathrm{p}$

Goring, D \& Raichlen, F. (1980). The generation of long waves in the laboratory. Proc. 17th Coastal Engineering Conference, ASCE, Vol. 1.pp 763 - 783.

Hull, P., Müller, G. 2002. An investigation of breaker heights, shapes and pressures. Ocean Engineering 29 pp. 59-79

Kaminski, M.L., H. Bogaert, 2009. Full Scale Sloshing Impact Tests. ISOPE conference. Osaka

Kirby, JT \& Dalrymple, RA (1986) An approximate model for nonlinear dispersion in monochromatic wave propagation models. Coastal Engineering 9.

Lamb, H. (1932) Hydrodynamics. 5th ed. Cambridge University Press, Cambridge.

Lugni C., Brocchini M., Faltinsen O.M., 2006, Wave impact loads: The role of the flip-through, Phys. of Fluids, 18 (12).

Minikin R.R., Wind, Waves and Maritime Structures, Charles Griffin a. Co. Ltd., London, 1950 (and 2nd edition 1963)

Oumeraci, H., Klammer, P., Partenscky, H.W., 1993. Classification of breaking wave loads on vertical structures. Journal of Waterway, Port, Coastal and Ocean Engineering, ASCE 119 (4), 381-397.

Oumeraci, H.; Kortenhaus, A.; Allsop,W.; de Groot,M. et al., 2001. Probabilistic Design Tools for Vertical Breakwaters, A.A. Balkema Publishers, Lisse.

Peregrine, D.H. 2003. Water-Wave Impact On Walls. Ann. Rev. Fluid Mech. 35:23-43

Stevenson T., The design and construction of harbours, 2 nd edition, Edinburgh, UK, 1874

Van den Boomgaard, M. (2003) Wave focussing in a laboratory flume. MSc thesis. Delft University of Technology. Dept. Geosciences and Hydraulic Engineering. 